\title{
The prevalence of Trichuris spp. infection in indoor and outdoor cats on St. Kitts
}

\author{
Jennifer K Ketzis ${ }^{1}$, Linda Shell ${ }^{2}$, Sarah Chinault ${ }^{3}$, Charles Pemberton ${ }^{4}$, Mary Mauldin Pereira ${ }^{1}$ \\ ${ }^{1}$ Department of Biomedical Sciences, Ross University School of Veterinary Medicine, Basseterre, St. Kitts and \\ Nevis, West Indies \\ ${ }^{2}$ Department of Clinical Sciences, Ross University School of Veterinary Medicine, Basseterre, St. Kitts and Nevis, \\ West Indies \\ ${ }^{3}$ Ross University School of Veterinary Medicine, Basseterre, St. Kitts and Nevis, West Indies \\ ${ }^{4}$ Diagnostic Laboratory, Ross University School of Veterinary Medicine, Basseterre, St. Kitts and Nevis, West \\ Indies
}

\begin{abstract}
Introduction: The present study was conducted to evaluate the prevalence of Trichuris spp. and other intestinal parasitic infections in owned cats on St. Kitts.

Methodology: The feces of 41 non-feral cats (23 indoor only; 18 indoor/outdoor) were examined for the presence of Trichuris spp. eggs.

Results: Nine (22\%) of the cats were positive for Trichuris spp. Prevalence of trichuriasis in indoor cats was $26.0 \%$ as compared to $16.7 \%$ in outdoor cats. Other parasites identified included Ancylostoma spp. (10\%; 4 cats), Toxocara cati (2\%; 1 cat), Platynosomum spp. (22\%; 9 cats), Mammomonogamus spp. (2\%; 1 cat) and coccidia (7\%; 3 cats).

Conclusion: On St. Kitts, indoor cats are as likely to have parasite infections as outdoor cats. Given the zoonotic potential of some of the identified parasites, periodical anthelmintic treatment should be provided to both indoor and outdoor cats.
\end{abstract}

Key words: Cats; Trichuris spp.; Caribbean; parasite prevalence.

J Infect Dev Ctries 2015; 9(1):111-113. doi:10.3855/jidc.5778

(Received 19 August 2014 - Accepted 27 November 2014)

Copyright (C) 2015 Ketzis et al. This is an open-access article distributed under the Creative Commons Attribution License, which permits unrestricted use, distribution, and reproduction in any medium, provided the original work is properly cited.

\section{Introduction}

Trichuriasis is a parasitic infection of the colon and cecum in dogs, cats, swine, humans and other animals caused by whipworms (Trichuris spp.). Trichuris trichiura, the human whipworm, T. suis, the pig whipworm, and T. vulpis, the dog whipworm, can cause diarrhea and inflammation of the cecum and colon [1-4]. T. suis is considered a zoonotic parasite and, while the zoonotic potential of $T$. vulpis is unclear, there is some evidence of an association of $T$. vulpis infection with Ancylostoma caninum, which is a zoonotic parasite [3].

In comparison to T. vulpis, T. suis and T. trichiura, much less is known about Trichuris serrata and Trichuris campanula, the two species of whipworms that can infect cats. Recent studies on the prevalence of parasites in cats in North America, Europe and Australia have shown low prevalence $(<2 \%)$ or no presence of Trichuris spp. [5-8]. As a result, whipworm infection in cats is not a primary differential diagnosis for cats with diarrhea. Also, unlike Trichuris in dogs, swine and humans, no anthelmintic is registered for the treatment of Trichuris spp. in cats.

For Caribbean and South American countries, the prevalence of Trichuris spp. infections in cats is believed to be higher [9] than reported elsewhere. On St. Kitts, an island in the West Indies bordered by the Caribbean Sea and the Atlantic Ocean, the prevalence of whipworm infections among non-owned/feral cats was determined to be $71 \%$ [10] based on egg identification from fecal samples. During necropsies of 7 cats (feral and non-feral) euthanized for various health issues at Ross University School of Veterinary Medicine (RUSVM), 2 to $>120$ adult Trichuris spp. were recovered from the cecum and colon (Ketzis, unpublished data 2013) and related pathological changes were documented in some of these cats.

This study was conducted to determine the prevalence of Trichuris spp. in owned cats on St. Kitts. 


\section{Methodology}

Cats owned by students, faculty and staff of RUSVM were enrolled in the study. To qualify for the study, each cat had to be owned and resident on St. Kitts for at least 4 months and not treated within the previous 4 months with a product containing an active ingredient known to be efficacious against Trichuris vulpis (such as fenbendazole, febantel, milbemycin oxime, moxidectin). Cats treated with endo- and ectoparasiticides with active ingredients not registered for the treatment of Trichuris vulpis (e.g., pyrantel pamoate, selamectin) were permitted in the study. Multi-cat households were included in the study, since many households are multi-cat and their inclusion was needed to be reflective of the population.

Each owner completed a brief questionnaire regarding age, breed, sex and origin (born on St. Kitts or imported) of the cat as well as history of anthelmintic treatment and housing (indoor vs outdoors). If the cat had any access to the outdoors, it was classified as an outdoor cat. When the owner did not know the exact date of birth, age was estimated based on how long the cat had been owned. Treatment dates with anthelmintics also were estimated for many of the cats.

Feces were collected by the owner and submitted to the diagnostic laboratory at RUSVM within 3 days of collection. Feces not submitted to the laboratory on the day of collection were stored in a home refrigerator until submission. In the laboratory, feces were stored at $2-8^{\circ} \mathrm{C}$ and samples were analyzed within 5 days of collection. Two grams of feces were analyzed using a standard double centrifugation procedure and Sheather's sugar solution $(\mathrm{spg}=1.27)$. In one case, due to a very high egg count, a modified McMaster was performed.
Exploratory statistical analyses were performed using a Mann-Whitney test (Minitab 16.2.4) or a Fisher's exact test (http://vassarstats.net/tab2x2.html).

\section{Results}

The feces of 41 cats from 31 households were analyzed. Eight multi-cat households were included in the study with 2 to 3 cats enrolled from each of these households. Twenty-three cats were indoor only and 18 were permitted outdoor. The majority of the cats, 27 of the $41(65.8 \%)$, were born on St. Kitts; of the remaining 14 cats, 13 were born in the U.S. and one in Ireland. The cats not native to St. Kitts had all been on St. Kitts for at least 7 months, providing sufficient time to be infected with parasites from St. Kitts. The average age of the cats was 52 months. All of the cats were spayed or neutered. Descriptive data for the cats are presented in Table 1.

Eggs of Trichuris spp. were seen in the feces of 9 $(22 \%)$ cats (Table 1). Although more indoor cats than outdoor cats were positive for Trichuris spp. infections (26\% vs $16.7 \%)$, this difference was not statistically significant $(\mathrm{p}>0.05)$. There were no statistically significant differences in origin, age and length of ownership for Trichuris spp. positive and negative cats. The mean Trichuris spp. eggs per gram of feces was 113 (geometric mean 25; range of 2-700).

Twenty of the 41 cats $(49 \%)$ in the study were infected with parasites. Of the $9(22 \%)$ cats positive for Trichuris spp., $5(56 \%)$ had no other parasite infections and 4 (44\%) had co-parasite infections: 2 (22\%) had Platynosomum spp.; 1 (11\%) had Platynosomum spp., Ancylostoma spp. and Mammomonogamus spp.; 1 (11\%) had coccidia. Eleven (34\%) of the 32 cats negative for Trichuris spp. were infected with other parasites: $5(16 \%)$ had

Table 1. Descriptive data for Trichuris spp. positive and negative cats

\begin{tabular}{llccc}
\hline & & & \multicolumn{2}{c}{ Trichuris spp. } \\
Negative cats \\
\end{tabular}


Platynosomum spp.; 1 (3\%) had Platynosomum spp. and Toxocara cati; 3 (9\%) had Ancylostoma spp. and; $2(6 \%)$ had coccidia.

\section{Discussion and conclusion}

This is the first study on intestinal helminth infections in owned cats in the Caribbean region. Both indoor and outdoor cats were infected to a high degree with intestinal helminths. The $22 \%$ prevalence of Trichuris spp. in owned cats found in this study is likely applicable to other Caribbean and South American countries, where high rates of infection have been noted in feral cat populations and case reports.

One potential source of infection for indoor only cats is residual eggs in the environment after the cat was dewormed when first acquired. The number of indoor cats with Platynosomum spp. infections might be due to the number of lizards inside of homes on St. Kitts.

While chronic inflammation of the mucosa of the cecum and colon has been associated with $T$. vulpis infections and intussusception [3], to date there are no studies documenting clinical signs or pathology of Trichuris spp. infections in cats. With concerns regarding the pathogenicity of Trichuris spp. in other hosts, an increased awareness of the potential clinical signs due to Trichuris spp. infections is needed. In addition, the zoonotic potential of Trichuris spp. in cats should be assessed, given the high prevalence. Since no anthelmintics are registered for the treatment of Trichuris spp. infections in cats, attention is needed in regards to the efficacy of routine anthelmintic treatments.

Our study is subject to limitations. First of all, the sample size was relatively low, and differences between characteristics thus did not show statistical significance. In addition, the cats sampled were all owned by one group (RUSVM faculty, staff and students); thus the results might not represent the overall owned cat population on St. Kitts. In addition, the prevalences found are likely to be underestimated since only one fecal sample per cat was analyzed and Trichuris spp. can be intermittent shedders.

In conclusion, our study documents a high prevalence of Trichuris spp. in owned cats on St. Kitts. Other parasite infections were also documented and the results emphasize the importance of conducting routine fecal examinations on all owned cats regardless of indoor or outdoor housing. Treatment and prevention programs should be tailored to the parasites in a given geographical location with special attention to those of zoonotic potential.

\section{Acknowledgements}

The authors would like to acknowledge Ross University School of Veterinary Medicine for funding this study.

\section{Authors' contributions}

Jennifer K. Ketzis: study design, enrollment of cats; data entry and review; statistical analysis, manuscript; Linda Shell: study design; IRB permission; enrollment of cats; review of manuscript; Sarah Chinault: enrollment of cats; study organization; data entry; Charles Pemberton: fecal analysis; review of manuscript; Mary Mauldin Pereira: enrollment of cats; review of manuscript

\section{References}

1. Yang HF, Wang CC, Hu CF, Hsieh CC, Lee HS, Chen SJ, Fan HC (2012) Importance of Considering Trichuris trichiura Infection in Infant Presenting with Acute and Substantial Bloody Diarrhea: A Case Report and Literature Review. J Med Sci 32: 309-312.

2. Pittman JS, Shepherd G, Thacker BJ, Myers GH (2010) Trichuris suis in finishing pigs: Case report and review. J Swine Health Prod 18: 306-313.

3. Traversa D (2011) Are we paying too much attention to cardio-pulmonary nematodes and neglecting old-fashioned worms like Trichuris vulpis? Parasites \& Vectors 4: 32.

4. Kirkova Z, Dinev I (2005) Morphological changes in the intestine of dogs, experimentally infected with Trichuris vulpis. Bulg J Vet Med 8: 239-243.

5. Barutzki D, Schaper R (2011) Results of parasitological examinations of faecal samples from cats and dogs in Germany between 2003 and 2010. Parasitol Res 109: S45-60.

6. De Santis AC, Raghavan M, Caldanaro RJ, Glickman NW, Moore GE, Lewis HB, Schantz PM, Glickman LT (2006) Estimated prevalence of nematode parasitism among pet cats in the United States. JAVMA 228: 885-92.

7. Gates MC, Nolan TJ (2009) Endoparasite prevalence and recurrence across different age groups of dogs and cats. Vet Parasitol 166: 153-8.

8. Ng BK, Kelly JD (1975) Anthropozoonotic helminthiases in Australasia: Part 3: - studies on the prevalence and public health implications of helminth parasites of dogs and cats in urban environments. Int J Zoonoses 2: 76-91.

9. Bowman DD, Hendrix CM, Lindsay DS, Barr SC (Eds.) (2002) Feline Clinical Parasitology. Iowa State University Press pp 348-350.

10. Krecek RC, Moura L, Lucas H, Kelly P (2010) Parasites of stray cats (Felis domesticus L., 1758) on St. Kitts, West Indies. Vet Parasitol 172: 147-9.

\section{Corresponding author}

Jennifer K. Ketzis

Department of Biomedical Sciences, Ross University School of

Veterinary Medicine, PO Box 334, Basseterre, St. Kitts and Nevis,

West Indies

Phone: +1 732-898-0055

Fax: +1 869-465-1203

Email:jketzis@rossvet.edu.kn

Conflict of interests: No conflict of interests is declared. 